\title{
Short Course Report for 1985
}

\author{
Vivienne Harwood Mattox \\ MRS Short Course Manager
}

The year 1985 was significant for the development of the MRS Short Course Program. Continuing a policy of expansion of the program to serve the needs of the MRS membership and materials research community, L. Ralph Dawson prepared a program of 12 short courses which were successfully presented over a period of two days at the 1985 MRS Fall Meeting. Five of the courses were new. For the first time, scholarship applications were invited from graduate students; seven individuals were selected to benefit from this new program.

In the Fall of 1985, an On-Site course program was developed. Two requests from industry for specific course topics to be held on their premises were received and one On-Site program was held in 1985. The On-Site course program is available to all organizations on an instructor-available basis. One of the benefits of the program is that the course content can be tailored to the needs of the requesting organization. In addition when held on-site, significant savings in terms of time and money are realized by the organization. For details concerning the availability of new and existing courses for this program, organizations should contact the MRS Short Course Manager, Vivienne Harwood Mattox, at (505) 294-9532.

A survey of the MRS membership conducted in the Fall identified some new course topics of interest to the membership. Every effort will be made to introduce some of these suggestions into the expanding course program in 1986 . The response from the membership for volunteers to assist in publicizing the course program within their organizations was impressive. This support from the membership will be utilized in 1986 and is greatly appreciated.

\section{Coming in 1986}

The five-day course program to be presented in conjunction with the 1986 MRS Spring Meeting in Palo Alto is summarized in this issue. The program consists of 16 courses, nine of which are new. One-, two- and three-day courses are offered, and discounted fees are available on certain groups of courses which can be taken in sequence. The brochure describing the Spring Meeting courses details who should attend and provides the course description and topical outline for each course day. Copies of the brochure can be obtained by writing or calling MRS Headquarters (telephone 412-367-3003).

In the future, core courses are anticipated which will provide an overview of certain topics. In addition short specialty-type courses will cover specific aspects of the core courses in depth. This approach will be applied to different areas of interest to materials scientists and engineers. A comprehensive curriculum of courses will be developed. Every effort will also be made to arrange for courses to precede or complement an MRS symposium on the same topic at MRS meetings.

Suggestions concerning the MRS Short Course Program are welcomed and should be directed to Vivienne Harwood Mattox at (505) 294-9532.

MIRIS

\section{NEW FROM MRS . . .}

\section{ON-SITE SHORT COURSES conducted at your own facilities or a nearby site}

The best way to keep your staff on top of their fields is through an on-going program of continuing education. One of the most efficient, cost-effective methods is to bring experts to your geographical location so that they can teach the latest techniques, discuss the newest research, and talk to your staff about the actual problems that they face daily.

The economic savings of On-Site programs are considerable, both in terms of time and budget. There are no travel fees or hotel costs for your staff, and attendees lose minimal time from their work.

MRS On-Site courses will be adapted to the specific needs of the sponsoring organization and the management will have an opportunity to interact with the instructor to discuss the course emphasis. Written requests from organizations will be handled on a first-come, first-served basis. For further details of courses which are available, prices and other information, write or call:

\author{
Vivienne Harwood Mattox \\ MRS Short Course Manager \\ Materials Research Society \\ 440 Live Oak Loop \\ Albuquerque, NM 87122 \\ (505) 294-9532
}




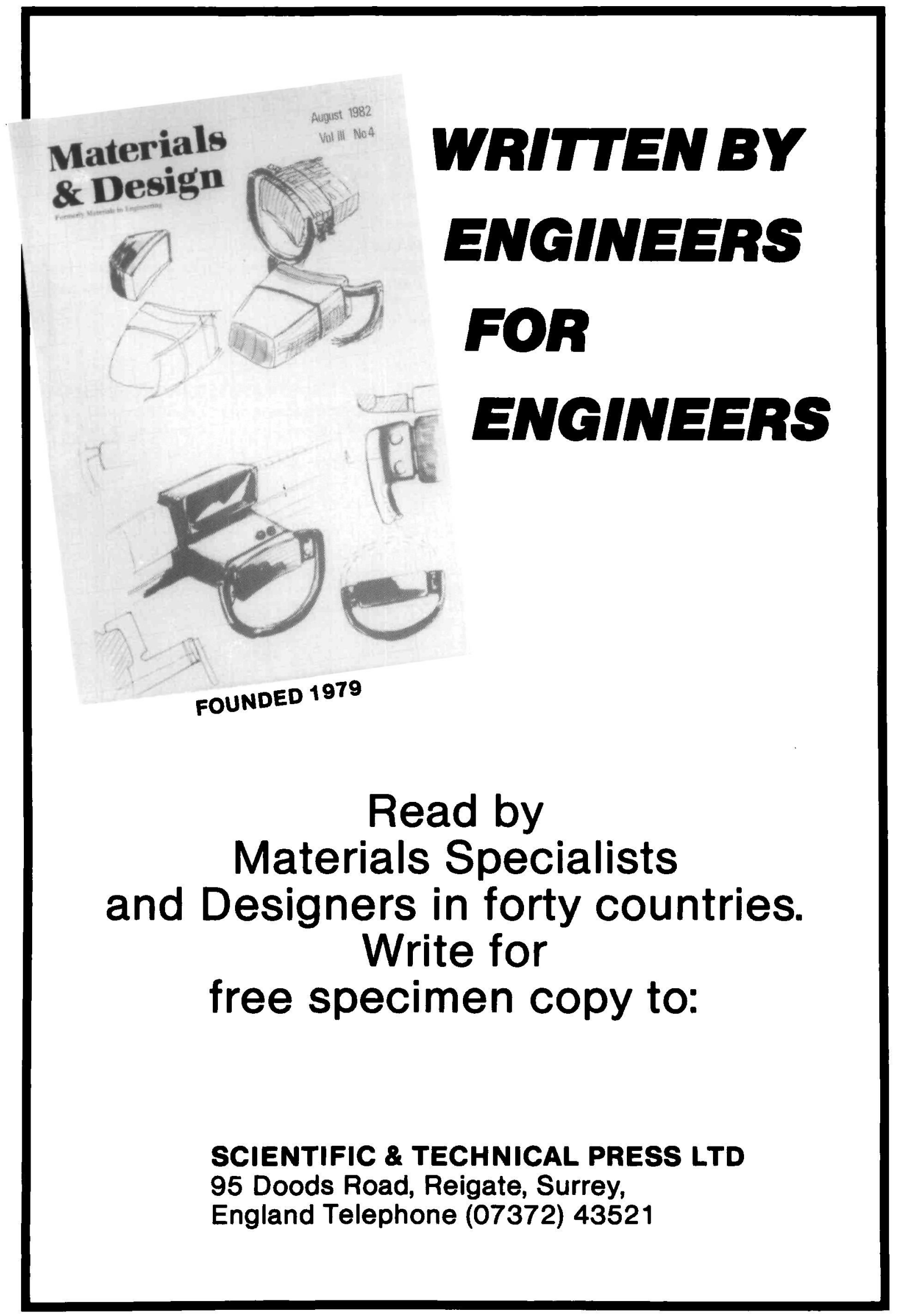




\section{PUBLICATIONS FROM 1985 MRS FALL MEETING \\ Prepublication Prices Effective Until March 30, 1986 \\ Publication: Spring 1986 \\ Volume 51}

BEAM-SOLID INTERACTIONS AND PHASE TRANSFORMATIONS

Editors: H. Kurz, Technical University Aachen; G. L. Olson, Hughes Research Laboratories; J.M. Poate, AT\&T Bell Laboratories Approximately $\mathbf{7 6}$ papers focus on laser-solid surface interactions, phase transitions and ion-solid interactions, laser-induced melting and exitation of semiconductors, laser-induced phase transformations in carbon and metals, ion beam-solid interactions, ion beam mixing and metastable phase formation.

Prepublication Prices: MRS Member $\mathbf{\$ 3 2}$ U.S. Nonmember- $\$ 38$ Foreign Nonmember- $\$ 43$

Regular Prices: MRS Member-\$37 U.S. Nonmember $-\$ 46$ Foreign Nonmember $-\$ 51$

\section{Volume 52}

\section{RAPID-THERMAL PROCESSING}

Editors: T.O. Sedgwick, IBM T.J. Watson Research Center; T.E. Siedel, J.C. Schumacher Co., B.Y. Tsaur, MIT Lincoln Laboratory Approximately 59 papers address implanted dopant activities and diffusion in silicon, defects and microstructure, equipment and device applications, rapid thermal processing for VLSI processing (panel discussion), silicides and oxides, compound semiconductors.

Prepublication Prices: MRS Member-\$30 U.S. Nonmember-\$36 Foreign Nonmember- $\$ 41$

Regular Prices: MRS Member-\$35 U.S. Nonmember-\$44 Foreign Nonmember-\$49

\section{Volume 53}

\section{SEMICONDUCTOR-ON-INSULATOR AND THIN FILM TRANSISTOR TECHNOLOGY}

Editors: A. Chiang, Xerox Palo Alto Research Center; M.W. Geis, MIT Lincoln Laboratory; L. Pfeiffer, AT\&T Bell Laboratories Approximately 67 papers focus on crystal growth and zone melting, epitaxy and SOS, implanted insulator, porous silicon, characterization of SOI thin films, polysilicon device and TFT, SOI devices and circuits/novel structures.

Prepublication Prices: MRS Member $\mathbf{\$ 3 2}$ U.S. Nonmember-\$38 Foreign Nonmember-\$43

Regular Prices: MRS Member-\$37 U.S. Nonmember-\$46 Foreign Nonmember-\$51

\section{Volume 54}

\section{THIN FILMS-INTERFACES AND PHENOMENA}

Editors: R.J. Nemanich, Xerox Palo Alto Research Center; P.S. Ho, IBM Watson Research Center; S.S. Lau, University of California Approximately 149 papers address silicide formation and properties, epitaxial layers, reaction kinetics, ion mixing, Schottky barriers, epitaxial silicide, ion-beam mixing, interface characterization, contact to III-Vs, compound semiconductor interfaces, VLSI technology and packaging, growth and analysis, oxides and nitrides.

Prepublication Prices: MRS Member-\$39 U.S. Nonmember-\$47 Foreign Nonmember- $\$ 52$

Regular Prices: MRS Member-\$45 U.S. Nonmember-\$55 Foreign Nonmember-\$59

\section{Volume 55 \\ BIOMEDICAL MATERIALS}

Editors: M.F. Nichols, University of Missouri; J.M. Williams, Oak Ridge National Laboratory; W. Zingg, University of Toronto Approximately 55 papers explore cardiovascular materials, opthalmic biomaterials, reconstruction materials for bone and teeth, materials for orthopaedics, biomaterials for electrodes, novel materials and techniques, materials problems in simulation and practice, science and technology of biomaterials.

Prepublication Prices: MRS Member-\$33 U.S. Nonmember-\$40 Foreign Nonmember-\$45

Regular Prices: MRS Member-\$37 U.S. Nonmember-\$46 Foreign Nonmember-\$51

\section{Volume 56 \\ LAYERED STRUCTURES AND EPITAXY}

Editors: M. Gibson, AT\&T Bell Laboratories; G.C. Osbourn, Sandia National Laboratories; R.M. Tromp, IBM Research Center Approximately 87 papers examine epitaxial semiconductor films, surfaces and ultrathin films, epitaxial silicides, quantum wells, strained layer superlattices, Ge/Si and noncrystalline superlattices, metal superlattices.

Prepublication Prices: MRS Member $\mathbf{\$ 3 4}$ U.S. Nonmember-\$41 Foreign Nonmember-\$46

Regular Prices: MRS Member-\$39 U.S. Nonmember-\$49 Foreign Nonmember-\$54

\section{Volume 57}

\section{PHASE TRANSITIONS IN CONDENSED SYSTEMS-EXPERIMENTS AND THEORY}

\section{Editors: G.S. Cargill III, IBM Corporation; F. Spaepen, Harvard University; K.N. Tu, IBM Research}

Approximately 37 papers explore nucleation, liquids, glasses, and glass formation; diffusion and defects, crystal growth and solid state transitions.

Prepublication Prices: MRS Member-\$32 U.S. Nonmember-\$38 foreign Nonmember-\$43

Regular Prices: MRS Member-\$37 U.S. Nonmember-\$46 Foreign Nonmember-\$51

\section{Volume 58}

\section{RAPIDLY SOLIDIFIED ALLOYS AND THEIR MECHANICAL AND MAGNETIC PROPERTIES}

Editors: B.C. Giessen, Barnett Institute; D.E. Polk, Office of Naval Research; A.I. Taub, G.E. Corporate Research and Development Approximately 73 papers address fundamentals and processing; structure and properties of amorphous, quasicrystalline and metastable crystalline alloys; rapidly solidified alloys and their mechanical properties; magnetic properties of rapidly solidified alloys.

Prepublication Prices: MRS Member-\$28 U.S. Nonmember-\$34 Foreign Nonmember-\$39

Regular Prices: MRS Member-\$33 U.S. Nonmember-\$41 Foreign Nonmember-\$46

\section{Volume 59}

\section{OXYGEN, CARBON, HYDROGEN, AND NITROGEN IN CRYSTALLINE SILICON}

Editors: J.W. Corbett, SUNY at Albany, J.C. Mikkelsen, Jr., Xerox Corporation; S.J. Pearton, AT\&T Bell Laboratories; S.J. Pennycook, Oak Ridge National Laboratory

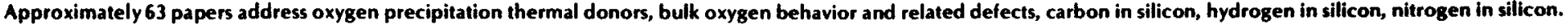

Prepublication Prices: MRS Member- $\$ 32$ U.S. Nonmember- $\$ 38$ Foreign Nonmember $-\$ 43$

Regular Prices: MRS Member-\$37 U.S. Nonmember-\$46 Foreign Nonmember-\$51 


\section{Volume 60}

\section{DEFECT PROPERTIES AND PROCESSING OF HIGH-TECHNOLOGY NONMETALLIC MATERIALS}

Editors: Y. Chen, Oak Ridge National Laboratory; W.D. Kingery, Massachusetts Institute of Technology; R.J. Stokes, Honeywell, Inc. Approximately 74 papers discuss advanced processing; lattice defects, interfaces and surfaces; surface modification; microwave processing; optical materials; mechanical properties.

Prepublication Prices: MRS Member-\$33 U.S. Nonmember-\$40 Foreign Nonmember $-\$ 45$

Regular Prices: MRS Member-\$38 U.S. Nonmember-\$48 Foreign Nonmember-\$53

\section{Volume 61}

\section{DEFECTS IN GLASSES}

Editors: Frank L. Galeener, Xerox Palo Alto Research Center; David L. Griscom, Naval Research Laboratory; Marvin J. Weber, Lawrence Livermore Laboratory

Approximately 44 papers explore silicon dioxide, intermediate range order, theory and modeling, short range order, impurities and dopants, chalcogenides, fibers and films, gel glasses, characterization.

Prepublication Prices: MRS Member $-\$ 28$ U.S. Nonmember- $\$ 34$ Foreign Nonmember $-\$ 39$

Regular Prices: MRS Member-\$33 U.S. Nonmember- $\$ 41$ Foreign Nonmember-\$46

\section{Volume 62}

\section{MATERIALS PROBLEM SOLVING WITH THE TRANSMISSION ELECTRON MICROSCOPE}

Editors: L.W. Hobbs, Massachusetts Institute of Technology; K.H. Westmacott, Lawrence Berkeley Laboratory; D.B. Williams, Lehigh University

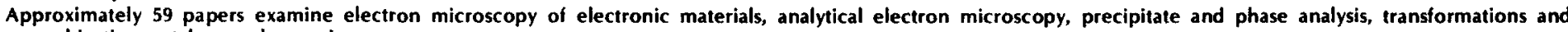
amorphization, catalysts and ceramics.

Prepublication Prices: MRS Member-\$33 U.S. Nonmember-\$40 Foreign Nonmember-\$45

Regular Prices: MRS Member-\$37 U.S. Nonmember-\$46 Foreign Nonmember-\$51

\section{Volume 63}

\section{COMPUTER-BASED MICROSCOPIC DESCRIPTION OF THE STRUCTURE \\ AND PROPERTIES OF MATERIALS}

Editors: J. Broughton, SUNY-Stonybrook; W. Krakow, IBM T.J. Watson Research Center; S.T. Pantelides, IBM T.J. Watson Research Center

Approximately 46 papers discuss applications in metals, electronic materials, alloys, glasses, and polymers, and theoretical studies.

Prepublication Prices: MRS Member $-\$ 30$ U.S. Nonmember $-\$ 36$ Foreign Nonmember $-\$ 41$

Regular Prices: MRS Member $-\$ 35$ U.S. Nonmember $-\$ 44$ Foreign Nonmember $-\$ 49$

\section{Volume 64}

\section{CEMENT-BASED COMPOSITES; STRAIN RATE EFFECTS ON FRACTURE}

Editors: S. Mindess, University of British Columbia; S.P. Shah, Northwestern

Approximately 24 papers explore strain-rate effects, cracking and strength at varying strain rates, dynamic effects.

Prepublication Prices: MRS Member-\$24 U.S. Nonmember-\$30 Foreign Nonmember-\$35

Regular Prices: MRS Member-\$29 U.S. Nonmember $-\$ 36$ Foreign Nonmember-\$43

\section{Volume 65}

\section{FLY ASH AND COAL CONVERSION BY-PRODUCTS; CHARACTERIZATION,UTILIZATION AND DISPOSAL II}

Editors: G.J. McCarthy, North Dakota State University; D.M. Roy, Pennsylvania State University

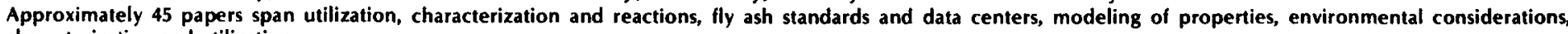
characterization and utilization.

Prepublication Prices: MRS Member-\$24 U.S. Nonmember-\$30 Foreign Nonmember-\$35

Regular Prices: MRS Member-\$29 U.S. Nonmember-\$36 Foreign Nonmember-\$43

\section{Volume 66}

\section{FRONTIERS IN MATERIALS EDUCATION}

\section{Editors: G.L. Liedl, Purdue University; L.W. Hobbs, Massachusetts Institute of Technology}

Approximately 23 papers discuss materials education-philosophy and future, industry-government interactions, ceramics, polymers, and problem areas, cross-discipline education.

Prepublication Prices: MRS Member $-\$ 24$ U.S. Nonmember $-\$ 30$ Foreign Nonmember $-\$ 35$

Regular Prices: MRS Member-\$29 U.S. Nonmember-\$36 Foreign Nonmember- $\$ 43$ AVAILABLE NOW...

\section{Extended Abstracts (EA-5) \\ BEAM-INDUCED CHEMICAL PROCESS}

Editors: R.J. von Gutfeld, IBM T.J. Watson Reseach Center; J.E. Greene, University of Illinois; I.Schlossberg, Air Force Office of Scientific Research

MRS Member $-\$ 5 \quad$ List $-\$ 7$

Extended Abstracts (EA-6)

FRACTAL ASPECTS OF MATERIALS

Editors: R.B. Laibowitz, IBM Reseach Center; B.B. Mandelbrot, Harvard University; D.E. Passoja, Passoja Inc.

MRS Member-\$5 List-\$7

Extended Abstracts (EA-7)

NONLINEAR OPTICAL MATERIALS

Editors: A.M. Glass, AT\&T Bell Laboratories; D.A.B. Miller, AT\&T Bell Laboratories; C.L. Tang, Cornell University

MRS Member-\$5 List-\$7 